\section{Sexual Health Is Paramount in the Counseling of Women at Risk for Breast or Ovarian Cancer Undergoing Risk-Reducing Surgery}

\author{
Jordan E. Rullo, PhD, ABPP, CST, and Sandhya Pruthi, MD
}

The greatest predictor of sexual dissatisfaction after risk-reducing salpingo oophorectomy (RRSO) is sexual dysfunction. ${ }^{1}$ However, $60 \%$ to $80 \%$ of patients with BRCA report that they did not receive any information about sexual health preoperatively. ${ }^{2}$ For women undergoing risk-reducing surgeries for elevated breast or ovarian cancer risk or hereditary predisposition, the impact on sexual health is one of the most influential factors when deciding to undergo these surgeries. ${ }^{3}$ Among BRCA carriers who have undergone RRSO, impact on sexual health encompasses 2 of the top 3 issues they wish they knew about before surgery. Specifically, hereditary breast or ovarian cancer (HBOC) carriers wish they knew the true impact surgery was going to have on their sexual health, and about the availability of sexual health counseling. ${ }^{2}$

Preoperative sexual health counseling is paramount for patients to be able to make a fully informed consent regarding surgical options. Comprehensive preoperative counseling has been found to increase surgery preparedness and decrease postsurgical sexual distress. ${ }^{4}$ However, most healthcare providers do not have the training to provide this counseling. As a result, postoperative symptoms that most often go undiscussed are those that relate to sexual health. ${ }^{2}$ One does not need to be a sexual health specialist to provide preoperative sexual health counseling. This article provides an outline of the preoperative sexual education essentials for counseling women at high risk for or carriers of $\mathrm{HBOC}$ mutations undergoing risk-reducing procedures.

\section{Impact of RRSO on Sexual Health}

Half of the most common postsurgical symptoms of RRSO directly impact sexual function, including vaginal dryness (52\%), change in sexual interest $(50 \%)$, and a self-reported change in sex life $(44 \%){ }^{2}$ Although for many women, hormone therapy can effectively treat common postsurgical symptoms of RRSO (eg, vaginal dryness), it does not effectively treat sexual dysfunction symptoms. ${ }^{5}$ Overall, sexual health and quality of life after RRSO for a premenopausal woman will be comparable to that of a postmenopausal woman. ${ }^{5}$

Vaginal dryness and genitourinary syndrome of menopause may lead to painful vaginal penetration. Patients need to be informed to not engage in painful sex, because this may lead to pelvic floor tension that exacerbates pain. In addition, waterbased lubricants and nonpainful, nonpenetrative sexual activities can be effective in managing symptoms.

With the loss of hormones and thus decreased blood flow to the genitals, women after RRSO report less genital sensitivity and greater difficulty with sexual arousal and orgasm..$^{5}$ Thus, sexual arousal and reaching orgasm take longer and are less intense. Vaginal sensitivity, in particular, may decrease with aging compared with clitoral sensitivity to vibration. Thus, the use of a vibrator for genital stimulation can enhance arousal and orgasm in women with compromised genital sensory thresholds.

Additionally, premenopausal women often report a decline in spontaneous sexual desire after oophorectomy. Although spontaneous desire declines, responsive desire may still be intact. ${ }^{6}$ Responsive desire differs from spontaneous desire in that it begins as a nonsexual state, wherein the woman encounters sexual stimuli (perhaps her

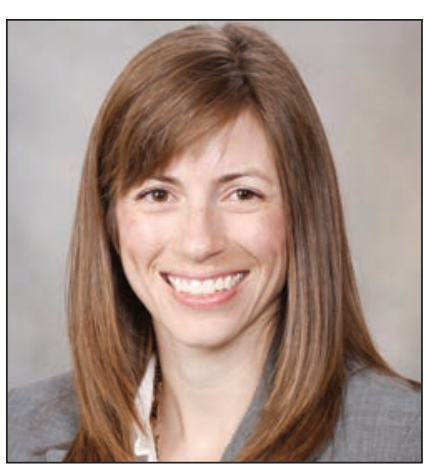

Jordan E. Rullo, PhD, ABPP,

CST

Jordan E. Rullo, PhD, ABPP,

CST, is a board certified Clinical Health Psychologist and Certified Sex Therapist through the American Association of Sex Educators, Counselors, and Therapists (AASECT). She is an Assistant Professor of Psychology, Department of Psychology and Psychiatry, Division of General Internal Medicine at Mayo Clinic in Rochester, Minnesota, and practices consultative health at the Women's Health Clinic at Mayo Clinic. Her areas of specialty include female and male sexual health concerns, sexual health related to menopause and cancer, transgender health, sexual orientation, and couple therapy. She trained at the Kinsey Institute at Indiana UniversityBloomington, the Centre for Addiction and Mental Health, and the University of Minnesota Program in Human Sexuality. She is a published author in peerreviewed journals on the topic of sexual health and has presented on sexual health at national and international conferences. 


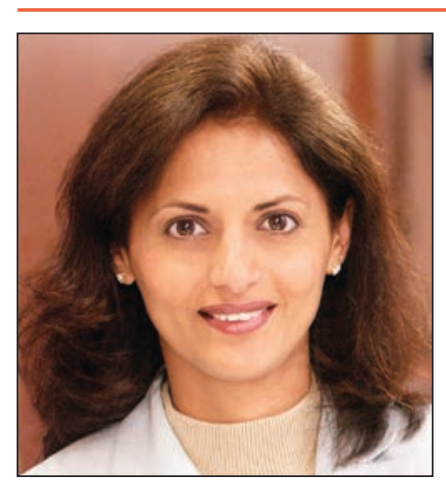

Sandhya Pruthi, MD

Sandhya Pruthi, MD, received her MD from the University of Manitoba, Canada, in 1990, and completed a Family Medicine residency at the Mayo Clinic in 1994. She was the Director of the Breast Diagnostic Clinic at Mayo Clinic in Rochester, Minnesota, from January 2002 to July 2010. She is a Consultant in the Department of General Internal Medicine and a Professor of Medicine.

Dr. Pruthi's research area is in breast cancer prevention. Her clinical expertise is the management of women at increased risk for breast cancer and providing preoperative counseling to women newly diagnosed with breast cancer. She is the Mayo Clinic principal investigator on several national breast cancer prevention and biomarker trials aimed at reducing the risk of breast cancer. She has collaborated in other women's health trials studying complementary and alternative therapies for the management of menopausal symptoms and improving the quality of life of breast cancer survivors.

Dr. Pruthi is the Chief Medical Editor and Associate Medical Director for Content Management and Delivery for Global Business Solutions. She is an Associate Medical Director for Development and physician partner in the Center for Innovation at Mayo Clinic. partner initiates sexual activity) and, once sexual activity begins, experiences sexual arousal, which leads to responsive sexual desire. ${ }^{6}$ With responsive desire, arousal precedes desire. Therefore, if a woman without desire is willing to become sexually aroused, responsive desire may follow. For premenopausal women after RRSO, responsive desire may become more prominent than spontaneous desire. Adapting to this change by creating a "new sexual normal" and learning ways to maximize responsiveness will be important for sexual satisfaction.

It is important to share with patients that the decline in sexual function with RRSO may not impact sexual satisfaction. For example, despite the changes in sexual function due to menopause, many postmenopausal women continue to experience sexual satisfaction. ${ }^{7}$ Additionally, relationship satisfaction has been shown to be the greatest predictor of change in sexual activity after RRSO. ${ }^{7}$ That is, women who reported high satisfaction in their relationships continued to be sexual, despite decline in sexual function. Therefore, a change in sexual function does not necessarily indicate a change in sexual satisfaction.

\section{Impact of Hysterectomy on Sexual Health}

Some women choose to undergo hysterectomy concurrent with RRSO. Although data on the impact of hysterectomy on sexual function are mixed, evidence shows that it may negatively impact sexual function and reduce genital sensation. ${ }^{8}$ Specifically, for women who need vaginal or cervical stimulation to experience orgasm, hysterectomy may compromise genital sensation and orgasm. However, for most women who experience orgasm through clitoral stimulation, hysterectomy should not impact orgasm. If orgasmic function has been compromised, clinicians can share with patients that many pathways exist to orgasm beyond vaginal and cervical stimulation. ${ }^{8}$

\section{Impact of Risk-Reducing Mastectomy on Sexual Health}

Of the BRCA carriers who have undergone risk-reducing mastectomy (RRM), more than a third report a decline in sexual well-being after surgery. ${ }^{9}$ Similar to with RRSO, most of the common postsurgical symptoms of RRM directly impact sexual function, including reduced or lack of sensation, unpleasant feeling in breast, decline in sexual pleasure, poor body image, and self-consciousness about body appearance. ${ }^{10}$ Women may find themselves disassociating from their breasts after surgery or reconstruction. Dissociation, or disconnecting oneself, is a way to cope with the trauma of one's breasts being poked, prodded, scarred, and stared at by numerous healthcare providers. In women for whom breast or nipple stimulation was necessary to achieve arousal and/or orgasm, this change in breast sensation can be a major loss.

Educating patients that mastectomy does not take away their sexuality is important. A woman after mastectomy will need to develop a new sexual normal, especially if their pathway to arousal orgasm (eg, nipple stimulation) has been compromised. In situations in which a patient has a partner, it is critical for the partner to help the patient achieve a new sexual normal. One way to begin this process is to encourage the patient to reconnect with her chest, breast, and/or body through scar tissue massage, mindfulness body scans, or partnered sensual touch exercises.

\section{Conclusions}

Preoperative sexual function education is an essential component of counseling women at high risk for or carriers of hereditary breast or ovarian mutations considering risk-reducing surgeries to guide them during the informed decision-making process. 
Healthcare providers can provide basic sexual health education and information on the impact of risk-reducing surgical procedures on overall sexual function and quality of life. This information will improve preparedness for surgery and decrease postsurgical sexual distress. Developing a new sexual normal, reconnecting with one's body, and (if partnered) allowing one's partner to reconnect, will be paramount in enhancing sexual health and satisfaction.

\section{References}

1. Robson M, Hensley M, Barakat R, et al. Quality of life in women at risk for ovarian cancer who have undergone risk-reducing oophorectomy. Gynecol Oncol 2003;89:281-287.

2. Campfield Bonadies D, Moyer A, Matloff E. What I wish I'd known before surgery: BRCA carriers' perspectives after bilateral salipingo-oophorectomy. Fam Cancer 2011;10:79-85.

3. Staton AD, Kurian AW, Cobb K, et al. Cancer risk reduction and reproductive concerns in female BRCA1/2 mutation carriers. Fam Cancer 2008;7:179-186.

4. Brotto LA, Branco N, Dunkley C, et al. Risk-reducing bilateral salpingo-oophorectomy and sexual health: a qualitative study. J Obstet Gynaecol Can 2012;34:172-178.

5. Finch A, Metcalfe KA, Chiang JK, et al. The impact of prophylactic salpingo-oophorectomy on menopausal symptoms and sexual function in women who carry a BRCA mutation. Gynecol Oncol 2011;121:163-168.

6. Faubion SS, Rullo JE. Sexual dysfunction in women: a practical approach. Am Fam Phys 2015;92:281-288.

7. Burri A, Hilpert $P$, Spector T. Longitudinal evaluation of sexual function in a cohort of pre- and postmenopausal women. J Sex Med 2015;12:1427-1435.

8. Komisaruk BR, Frangos E, Whipple B. Hysterectomy improves sexual response? Addressing a crucial omission in the literature. J Minim Invasive Gynecol 2011;18:288-295.

9. Razdan SN, Patel V, Jewell S, McCarthy CM. Quality of life among patients after bilateral prophylactic mastectomy: a systematic review of patient-reported outcomes. Qual Life Res 2016;25:1409-1421.

10. Gopie JP, Mureau MA, Seynaeve C, et al. Body image issues after bilateral prophylactic mastectomy with breast reconstruction in healthy women at risk for hereditary breast cancer. Fam Cancer 2013;12:479-487. 